\title{
Analysis of the Separation Principle of Kinematic Errors from the Rotation Axis in Manufacturing Equipment
}

\author{
Ya Zhang ${ }^{1}$, Lin Zhang ${ }^{2}$ \\ 1. Zhejiang University of Science and technology, Hangzhou, 310027, China \\ 2. Yangzhou Polytechnic College, Yangzhou, 225127, China \\ 1yazhang1982@126.com; ${ }^{2} 330213049 @ q q . c o m$
}

\section{Keywords: Kinematic Errors; Rotation Axis; Machine Tool}

Abstract. Machining tests were used to identify the kinematic errors of the rotary axes in machine tools. The key to design the machining tests is analyzing the relationship of the kinematic errors of the machine tool and the machining errors of the test workpiece. By adopting the error-sensitive direction vector, the relationship of the machining errors of the test workpiece and the kinematic errors of machine tool rotation axis was analyzed. Then the error-sensitive direction vector is adopted to separate the individual kinematic error of the rotation axis. This analysis method can support a foundation and reference for the machining tests design to separate the rotation axis in all types of manufacturing equipment.

\section{Introduction}

Multi-axis NC machine tools are suitable for manufacturing complicated space curved surface or the cavity surface. They were widely used in manufacturing the wings, impellers in the modern manufacturing industry. At present, the development of multi-axis CNC machine tools has become an urgent need for industrial production. However, due to the influence of the superposition of rotary axes and the straight axis of multi-axis NC machine tool, the kinematic chain of the machine tool is longer than three-axis machine tool. So the machining accuracy of multi-axis NC machine tool is usually lower than three-axis machine tool. Therefore, how to improve the machining accuracy has become the main object of the research of the multi-axis CNC machine tool in recent years.

The accuracy of motion is the most basic index for the development of high-precision multi-axis machine tool, and the key of improving the accuracy of the machine tool is to make rapid and accurate identification of the kinematic errors.

\section{Separation methods of the kinematic errors in the rotation axis of multi-axis machine tool table}

Error estimation of the machine tool includes two main directions: direct measurement methods and indirect measurement methods. Indirect measurement methods were also called error identification methods [1].

Direct measuring method indicates measuring single kinematic error in a measurement process. Single kinematic error of the rotary axis in multi-axis machine tool can be directly measured by laser interferometer[2].However, due to the superimpose of the kinematic errors of the machine tool, it is difficult to identify the kinematic errors of the rotation axis multi-axis machine tool.

Indirect measurements primarily detect the position of tool center point ( TCP ) of the machine tool, rather than the individual error of a moving part. In earlier studies, indirect measurements were only used for rapid detection of total machine performance. In recent years, many researchers have proposed the application of indirect measurement method to establish machine tool kinematic model and identify the kinematic error in single part of the machine tool. This is the error identification technology. The existing identification methods of the kinematic error of multi-axis machine tool rotary axis almost adopted the special instruments, such as telescoping double ball bar [3,4], R-test [5,6], and 
so on. They have the disadvantages of computational complexity, time-consuming of operation, high cost, and so on. The versatility of these methods is not strong, too. To meet the actual need of the machine tool users and manufacturers, machining tests were designed to identify the kinematic errors of rotary axis of multi-axis machine tool table. The key to design the machining tests is analyzing the relationship of the kinematic errors of the machine tool and the machining errors of the test workpiece. This paper focus on the principle of the error separation, .

\section{Machining tests}

The rotation axis of multi-axis table is also called $\mathrm{C}$-axis. There are three linear displacement errors ${ }^{C} \delta_{x}^{\prime},{ }^{C} \delta_{y}^{\prime},{ }^{C} \delta_{z}^{\prime}$ and three angular errors ${ }^{C} \xi_{x},{ }^{C} \xi_{y},{ }^{C} \xi_{z}$ in C-axis. Because ${ }^{C} \delta_{z}^{\prime}$ can be eliminated by tool setting and workpiece localization, and the kinematic error ${ }^{C} \xi_{z}$ in the direction of the output of $\mathrm{C}$-axis rotation has no influence of the machining errors. Therefore, only four kinematic errors of $\mathrm{C}$-axis need to be identified. The steps of the machining tests of $\mathrm{C}$-axis are as follows:

(1) Install the workpiece in the initial position. At this time, the $Y$ axis remains stationary, the A axis remains horizontally and stationary, the workpiece is fit at the angle of 0 degree in the rotation axis ( $\mathrm{C}$ axis) in $+X$ direction, and the distance from the workpiece center is $L$.

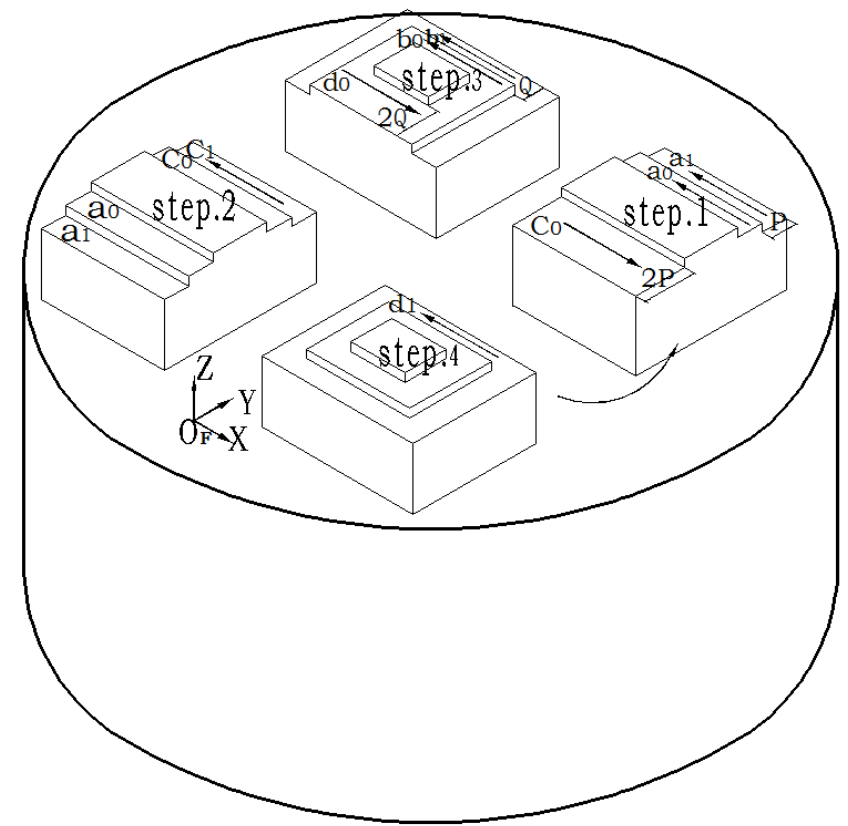

Figuer.1 Machining tests to separate the kinematic errors in C-axis

(2) Machining the measurement datum plane

Firstly, cut a plane on both sides of the workpiece in $+Y$ direction. Then, cut a plane on both sides of the workpiece in $+X$ direction. The width is $2 l$, the cutting depth is $h$. Thus, four intersecting lines were produced. They are $a_{0} 、 b_{0} 、 c_{0} 、 d_{0}$ 。 The distance between $a_{0}$ and $c_{0}$ is $P_{0}$. The distance between $b_{0}$ and $d_{0}$ is $Q_{0}$.

(3) Machining the object plane

After machining the reference plane, return the tool to the machining start point to eliminate the effect of motion error on the object plane. At the angle of 0 degree in the rotation axis, cut a plane on the edge of the workpiece in $+Y$ direction. So, an intersecting line was produced. It is $a$. At the angle of 90 degree, 180 degree and 270 degree in the rotation axis, cut a plane on the edge of the workpiece in $+Y$ direction. So, three intersecting lines were produced. They are $b, c, d$. Where, the distance between the horizontal plane near the intersecting line a and the horizontal plane near the intersecting line $c$ is P. The distance between the level plane near the intersecting line $a$ and the level plane near the intersecting line $c$ is $H_{c a}$. The distance between the horizontal plane near the intersecting line $b$ and the horizontal 
plane near the intersecting line $d$ is $Q$. The distance between the level plane near the intersecting line $b$ and the level plane near the intersecting line $d$ is $H_{b d}$.

\section{Analysis of the Separation Principle of Kinematic Errors}

To analyze the relationship of the kinematic error of $\mathrm{C}$-axis and the machining error of the test workpiece, the error-sensitive direction vector[7] is adopted to seperate the individual kinematic error. For example, $r_{1}=\left[\begin{array}{lll}1 & 0 & 0\end{array}\right]$ represents that the error sensitive direction is $+X$. It can be adopted to separate the single error in the direction of $+X$. Therefore, $r_{1}=\left[\begin{array}{lll}1 & 0 & 0\end{array}\right]$ corresponds to the kinematic error of ${ }^{C} \delta_{x}^{\prime}$. Similarly, $r_{2}=\left[\begin{array}{lll}0 & 1 & 0\end{array}\right]$ represents that the error sensitive direction is $+Y$. It corresponds to the kinematic error of ${ }^{C} \delta_{y}^{\prime} . r_{3}=\left[\begin{array}{lll}0 & 0 & -1\end{array}\right]$ represents that the error sensitive direction is $-Z$. It corresponds to the kinematic error of ${ }^{C} \xi_{y}(\gamma)$ and ${ }^{C} \xi_{x}(\gamma)$. According to the kinematic principle of multi-axis machine tool, the separation principle of kinematic errors is analyzed as follows:

$$
\begin{aligned}
& \Delta P=\left[\begin{array}{c}
L C 180^{\circ}+{ }^{C} \delta_{\delta_{x}}^{\prime}\left(180^{\circ}\right) \\
L S 180^{\circ}+{ }^{C} \delta_{y}\left(180^{\circ}\right) \\
L^{C} \xi_{x}\left(180^{\circ}\right) S 180^{\circ}-L^{C} \xi_{y}\left(180^{\circ}\right) C 180^{\circ}
\end{array}\right]\left[\begin{array}{lll}
1 & 0 & 0
\end{array}\right]-\left[\begin{array}{c}
L C 0^{0} \\
L S 0^{0} \\
0
\end{array}\right]\left[\begin{array}{lll}
1 & 0 & 0
\end{array}\right] \\
& \Delta Q=\left[\begin{array}{c}
L C 270^{0}+{ }^{C} \delta_{x}^{\prime}\left(270^{0}\right) \\
L S 270^{\circ}+{ }^{C} \delta_{y}\left(270^{0}\right) \\
L^{C} \xi_{x}\left(270^{0}\right) S 270^{0}-L^{C} \xi_{y}\left(270^{0}\right) C 270^{\circ}
\end{array}\right]\left[\begin{array}{lll}
0 & 1 & 0
\end{array}\right]- \\
& {\left[\begin{array}{c}
L C 90^{\circ} \\
L S 90^{0} \\
L^{C} \xi_{x}\left(90^{\circ}\right) S 90^{\circ}-L^{C} \xi_{y}\left(90^{\circ}\right) C 90^{\circ}
\end{array}\right]\left[\begin{array}{lll}
0 & 1 & 0
\end{array}\right]} \\
& \Delta h_{a c}=\left[\begin{array}{c}
L C 180^{\circ}+{ }^{C} \delta_{r}^{\prime}\left(180^{\circ}\right) \\
L S 180^{\circ}+{ }^{C} \delta_{y}\left(180^{\circ}\right) \\
L^{C \xi_{x}}\left(180^{0}\right) S 180^{\circ}-L^{C} \xi_{y}\left(180^{\circ}\right) C 180^{0}
\end{array}\right]\left[\begin{array}{lll}
0 & 0 & -1
\end{array}\right]- \\
& {\left[\begin{array}{c}
L C 0^{0} \\
L S 0^{0} \\
L^{C \xi_{x}}\left(0^{0}\right) S 0^{0}-L^{C \xi_{y}}\left(0^{0}\right) C 0^{0}
\end{array}\right]\left[\begin{array}{lll}
0 & 0 & -1
\end{array}\right]}
\end{aligned}
$$




$$
\begin{gathered}
\Delta h_{b d}=\left[\begin{array}{c}
L C 270^{\circ}+{ }^{C} \delta_{r_{x}}^{\prime}\left(270^{\circ}\right) \\
L S 270^{\circ}+{ }^{C} \delta_{y}\left(270^{\circ}\right) \\
L^{C} \xi_{x}\left(270^{\circ}\right) S 270^{\circ}-L^{C} \xi_{y}\left(270^{\circ}\right) C 270^{0}
\end{array}\right]\left[\begin{array}{lll}
0 & 0 & -1
\end{array}\right]- \\
L C 90^{0} \\
L 90^{\circ} \\
{\left[\begin{array}{c}
C \xi_{x}\left(90^{\circ}\right) S 90^{\circ}-L^{C} \xi_{y}\left(90^{\circ}\right) C 90^{0}
\end{array}\right]\left[\begin{array}{lll}
0 & 0 & -1
\end{array}\right]}
\end{gathered}
$$

Where, According to Equation (1), ${ }^{C} \delta_{x}^{\prime}\left(180^{\circ}\right)$ can be obtained. ${ }^{C} \delta_{x}^{\prime}\left(180^{0}\right)$ represents the in the direction of $+X$, when the rotation angle is 180 degree.

According to Equation (2), (3), (4), ${ }^{C} \delta_{y}^{\prime}\left(270^{\circ}\right)-{ }^{C} \delta_{y}^{\prime}\left(90^{0}\right),{ }^{C} \xi_{y}\left(180^{0}\right),{ }^{C} \xi_{x}\left(270^{0}\right)-{ }^{C} \xi_{x}\left(90^{0}\right)$ can be obtained.

Thus, four kinematic errors of C-axis were separated when the rotary angle of C-axis is 180 degrees.

\section{Conclusions}

Manufacturing equipment with a rotary table usually has the rotary axis superimposed with the linear axis together. Therefore, it is difficult to separate the kinematic errors of the rotation axis. Machining tests were used to identify the kinematic errors of the rotation axis. First of all, the relationship of the kinematic errors of the machine tool and the machining errors of the test workpiece should be established according to the kinematic principle. Then, some appropriate mathematical tools, such as error-sensitive direction vector, should be adopted in the analysis. At last, through matrix calculation, individual kinematic error of the rotation axis can be separated from the machining errors of the test workpiece. This analysis method can support a foundation and reference for the machining tests design to separate the rotation axis in all types of manufacturing equipment, such as machining robot, parallel kinematic machine, and so on.

\section{Acknowledgements}

This work was financially supported by Startup Foundation of Zhejiang University of Science and Technology (F701102H02).

\section{References}

[1] Soichi Ibaraki and Wolfgang Knapp. Indirect Measurement of Volumetric Accuracy for Three-Axis and Five-Axis Machine Tools: A Review. Int. J. of Automation Technology, 2012. 6(2): p.110-124.

[2] H. Schwenke, W. Knapp, H. Haitjema, A. Weckenmann, R. Schmitt, and F. Delbressine, Geometric error measurement and compensation of machines -An update. CIRP Annals - Manufacturing Technology, 2008. 57(2): p.560-575.K. Inasaki, H. Kishinami, N. Sakamoto, Y. Sugimura, F. Takeu-chi, Shape generation theory for machine tools, Yokendo, (1997)95-100.

[3] ISO/CD 10791-6, Test conditions for machining centres - Part 6: Accuracy of feeds, speeds and interpolations, 2011.

[4] S. Zargarbashi and J. Mayer, Assessment of machine tool trunnion axis motion error, using magnetic double ball bar. Int. J. of Machine Tools and Manufacture, 2006. 46(14): p.1823-1834. 
[5] B. Bringmann and W. Knapp, Model-based 'Chase-the-Ball' calibration of a 5-axis machining center. CIRP Annals - Manufacturing Technology, 2006. 55(1): p.531-534.

[6] Weikert, S., R-test, a new device for accuracy measurements on five axis machine tools. Cirp Annals-Manufacturing Technology, 2004. 53(1): p. 429-432.

[7] Zargarbashi SHH, Mayer JRR. Assessment of machine tool trunnion axis kinematic error, using magnetic double ball bar. Int J Mach Tools Manuf, 2006. 46(14):1823-1834 